\title{
Intercellular adhesion molecule 1 rs5498 polymorphism is associated with the risk of myocardial infarction
}

\author{
Pengfei Hu${ }^{1, *}$, Tao Dai ${ }^{2, *}$, Weiwei Yu', Ying Luo ${ }^{1}$ and Shuwei Huang ${ }^{1}$ \\ ${ }^{1}$ Department of Cardiology, The Second Affiliated Hospital of Zhejiang Chinese Medical University, Hangzhou, Zhejiang, China \\ ${ }^{2}$ Department of Cardiology, Yuhuangding Hospital Affiliated to Qingdao University, Yantai, Shandong, China \\ *These authors contributed equally to this work
}

Correspondence to: Shuwei Huang, email: shuweihuang163@163.com

Keywords: ICAM-1, rs5498, myocardial infarction, meta-analysis

Received: March 20, $2017 \quad$ Accepted: April 17, $2017 \quad$ Published: April 29, 2017

Copyright: Hu et al. This is an open-access article distributed under the terms of the Creative Commons Attribution License 3.0 (CC BY 3.0 ), which permits unrestricted use, distribution, and reproduction in any medium, provided the original author and source are credited.

\section{ABSTRACT}

Several studies addressed the association between Intercellular adhesion molecule 1 (ICAM-1) rs5498 polymorphism and Myocardial Infarction (MI) risk. However, they addressed conflicting findings. Therefore, the aim of this study was to explore whether ICAM-1 gene rs5498 polymorphism plays an important role in modifying the risk of MI. A meta-analysis was conducted on the association between ICAM-1 rs5498 polymorphism and MI. 12 eligible studies involving 1,696 cases and 3,039 controls were included in the meta-analysis. Meta-analysis revealed that ICAM-1 rs5498 polymorphism showed a strongly positive correlation with MI and could be viewed as a protective factor for MI. Furthermore, subgroup analysis according to ethnicity indicated that ICAM-1 rs5498 polymorphism decreased the risk of MI among Caucasian and Asian populations. In conclusion, ICAM-1 rs5498 polymorphism was associated with the decreased risk of MI. Larger sample size studies with more diverse ethnic populations are needed to confirm these findings.

\section{INTRODUCTION}

Cardiovascular disease (CVD), including myocardial infarction (MI), is the number one cause of death [1]. Suspected MI is a common reason for emergency hospital attendance and admission [2]. It has been suggested that biomarkers of inflammation, such as increased blood homocysteine [3], C-reactive protein [3], and cytokine levels [4], may be new risk factors for CVD. However, these biomarkers are poorly specific and susceptible. Inherited gene variants are less influenced by environment factors, and may be a better marker of individual MI susceptibility [5].

Intercellular adhesion molecule 1 (ICAM-1) is the member of the adhesion immunoglobulin super family that maps to chromosome 19 p13.2-p13.3 codes for 505 amino acids with five extracellular domains [6]. ICAM-1 is implicated in neutrophil and monocyte-endothelial cell adhesion, processes contributing to myocardial neutrophil infiltration and microvascular coronary slow flow, both viewed as important to the pathophysiologic responses in acute myocardial infarction (AMI) [7]. Patients with stable angina pectoris who developed MI had elevated serum levels of soluble ICAM-1, indicating increased inflammatory activity [8]. Kamijikkoku et al. found that a persistent increase in plasma soluble ICAM-1 levels may indirectly implicate vascular inflammation, which could predict the risk of early coronary restenosis after emergency angioplasty in patients with AMI [9]. Moreover, Niessen et al. identified that inhibition of ICAM-1 expression in the heart dramatically reduces infarct size [10].

Recently, several studies addressed the association between ICAM-1 gene rs5498 polymorphism and MI risk. However, they yielded contradictory and inconclusive findings [11-22]. Thus, we undertook this meta-analysis to evaluate whether ICAM-1 rs5498 polymorphism is associated with MI risk.

\section{RESULTS}

Characteristics of the included studies

261 articles were retrieved after initial search, and 45 full articles were identified for possible inclusion. 33 articles were excluded due to the following reasons: 
9 investigated other polymorphisms; 3 did not provide detailed genotyping data; 14 studied coronary artery disease (CAD); 3 were reviews; 3 were meta-analyses and 1 was not case-control study. In total, 12 eligible studies involving 1,696 cases and 3,039 controls were included. Selection for eligible studies in this meta-analysis was presented in Figure 1. The detailed characteristics of all the selected studies were summarized in Table 1.

\section{Quantitative synthesis of data}

A summary of the meta-analysis findings of the associations between the ICAM-1 rs5948 polymorphism and $\mathrm{MI}$ is provided in Table 2 and Table 3. As shown in Table 2, ICAM-1 rs5948 polymorphism was associated with the decreased risk of MI (G vs. A: OR, 0.60; 95\% CI, 0 0.47-0.76, $P<0.001$; Figure 2). Stratification analyses were conducted according to ethnicity, source of controls (SOC) and Hardy-Weinberg Equilibrium (HWE) status. In Analysis after stratification by ethnicity, significant association between ICAM-1 rs5948 polymorphism and MI risk was observed not only among Caucasian populations $(\mathrm{AG}+\mathrm{GG}$ vs. $\mathrm{AA}: \mathrm{OR}=0.66,95 \% \mathrm{CI}$ 0.46-0.92, $P<0.001$, Figure 3) but also among Asian populations $(\mathrm{AG}+\mathrm{GG}$ vs. $\mathrm{AA}: \mathrm{OR}=0.48,95 \% \mathrm{CI}$ $0.38-0.61, P<0.001$, Table 3). Stratification analysis of SOC found that ICAM-1 rs5948 polymorphism decreased the risk of MI among hospital-based controls (AG+GG vs. AA, Figure 4), but not among populationbased controls (Table 3 ). One study did not conform to HWE, but stratification analysis of HWE status revealed the conclusions of the remaining studies did not significantly alter (GG vs. AA+AG, Figure 5), suggesting that the results of ICAM-1 rs5498 polymorphism were trustworthy.

Sensitivity analysis was conducted to evaluate the effect of each study on the pooled ORs by omitting each study in turn. The pooled ORs were not affected by excluding any study (AG+GG vs. AA, Figure 6). Meanwhile, the potential publication bias (G vs. A, $P_{\text {begg }}=0.675$ and $P_{\text {egger }}=0.548 ; \mathrm{AG}+\mathrm{GG}$ vs. AA, $P_{\text {begg }}^{\text {begg }}=0.815$ and $\left.P_{\text {egger }}=0.593\right)$ was analyzed by performing both of the Begg's and Egger's tests (AG vs. AA, Figure 7). We found no obvious publication bias in this meta-analysis.

\section{DISCUSSION}

In this current meta-analysis, ICAM-1 gene rs5498 polymorphism showed a significant association with the decreased risk of MI. Stratification analysis of ethnicity found that rs5498 polymorphism decreased the risk of MI among Caucasians and Asians.

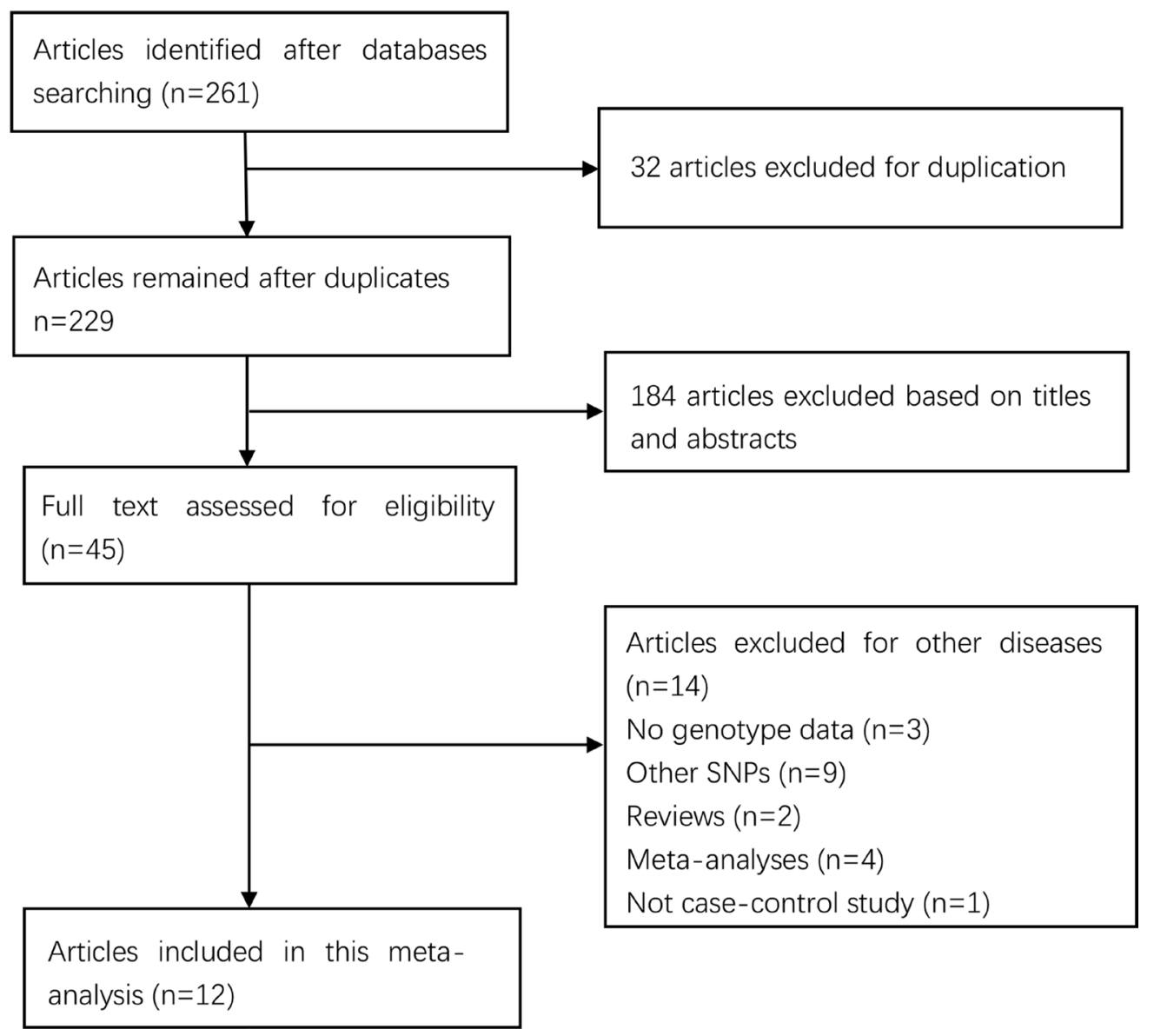

Figure 1: Selection for eligible citations included in this meta-analysis. 
Table 1: Characteristics of included studies

\begin{tabular}{lcccccccccccc}
\hline Surname & Year & SOC & Nationality & Ethnicity & $\begin{array}{c}\text { Number } \\
\text { of cases }\end{array}$ & \multicolumn{3}{c}{$\begin{array}{c}\text { Number of } \\
\text { controls }\end{array}$} & & HWE & NOS \\
\hline & & & & & AA & AG & GG & AA & AG & GG & \\
\hline Nasibullin & 2016 & HB & Russian & Caucasian & 101 & 152 & 62 & 90 & 145 & 51 & Y & 6 \\
Gazi & 2014 & HB & Turkey & Caucasian & 12 & 27 & 9 & 8 & 33 & 26 & Y & 7 \\
Burazynska & 2012 & HB & Poland & Caucasian & 69 & 44 & 5 & 272 & 379 & 173 & Y & 8 \\
Liu & 2011 & HB & China & Asian & 105 & 49 & 10 & 130 & 138 & 34 & Y & 6 \\
Mohamed & 2010 & HB & Egypt & Caucasian & 17 & 28 & 28 & 2 & 11 & 37 & Y & 7 \\
Sakowicz & 2010 & PB & Poland & Caucasian & 54 & N/A & $106 *$ & 48 & 69 & 14 & Y & 8 \\
Aminian & 2007 & HB & Iran & Caucasian & 42 & 77 & 33 & 36 & 69 & 35 & Y & 8 \\
Podgoreanu & 2006 & HB & America & Caucasian & 14 & 26 & 12 & 50 & 177 & 155 & Y & 8 \\
Milutinovic & 2006 & HB & Slovenia & Caucasian & 47 & 72 & 33 & 65 & 109 & 41 & Y & 6 \\
Wei & 2005 & HB & China & Asian & 76 & 35 & 7 & 101 & 103 & 26 & Y & 6 \\
Wang & 2005 & HB & China & Asian & 96 & 61 & 8 & 91 & 90 & 18 & Y & 7 \\
Jiang & 2002 & PB & German & Caucasian & 63 & 78 & 38 & 60 & 66 & 87 & N & 7 \\
\hline
\end{tabular}

SOC, source of controls; PB, population-based controls; HB, hospital-based controls; NOS, Newcastle-Ottawa Scale; HWE: Hardy-Weinberg equilibrium.

*The Combined number of AG and GG genotypes; N/A: not available.

Study
ID

Figure 2: ORs and $95 \%$ CIs from individual studies and pooled data for the association between ICAM-1 gene rs5948 polymorphism and MI in all subjects (G vs. A). 
Table 2: Meta-analysis of association between ICAM-1 rs5498 polymorphism and Myocardial infarction

\begin{tabular}{lccccc}
\hline \multicolumn{1}{c}{ Comparison } & OR(95\%CI) & $\boldsymbol{P}$-value & P for heterogeneity & $\mathbf{I}^{\mathbf{2}} \mathbf{( \% )}$ & Model \\
\hline G vs. A & $\mathbf{0 . 6 0}(\mathbf{0 . 4 7 , 0 . 7 6 )}$ & $<0.001$ & $<0.001$ & 81.9 & Random \\
AG+GG vs. AA & $\mathbf{0 . 6 0}(\mathbf{0 . 4 6 , 0 . 7 9 )}$ & $<0.001$ & $<0.001$ & 71.7 & Random \\
GG vs. AA + AG & $\mathbf{0 . 5 1}(\mathbf{0 . 3 5 , 0 . 7 5 )}$ & $<0.001$ & $<0.001$ & 73.3 & Random \\
GG vs. AA & $\mathbf{0 . 4 1}(\mathbf{0 . 2 6 , 0 . 6 5 )}$ & $<0.001$ & $<0.001$ & 75.0 & Random \\
AG vs. AA & $\mathbf{0 . 6 6 ( 0 . 5 2 , 0 . 8 3 )}$ & $<0.001$ & 0.014 & 55.1 & Random \\
\hline
\end{tabular}

*Bold values are statistically significant $(P<0.05)$.

ICAM-1 is a transmembrane glycoprotein in the immunoglobulin superfamily, which plays an important role in cell adhesion and signal transduction [23]. Ley et al. found that ICAM-1 is responsible for formation, growth and rupture of atheroma [24]. A circulating or soluble form of ICAM-1 with elevated levels were also observed in patients with atherosclerosis and heart failure [25]. A host of studies attached importance to assess the possibility that ICAM-1 gene polymorphism may become a new biomarker for MI [11-22]. Eight studies found that ICAM-1 rs5498 polymorphism was associated with a significantly increased risk of MI [12, 14, 16-18, 20-22].

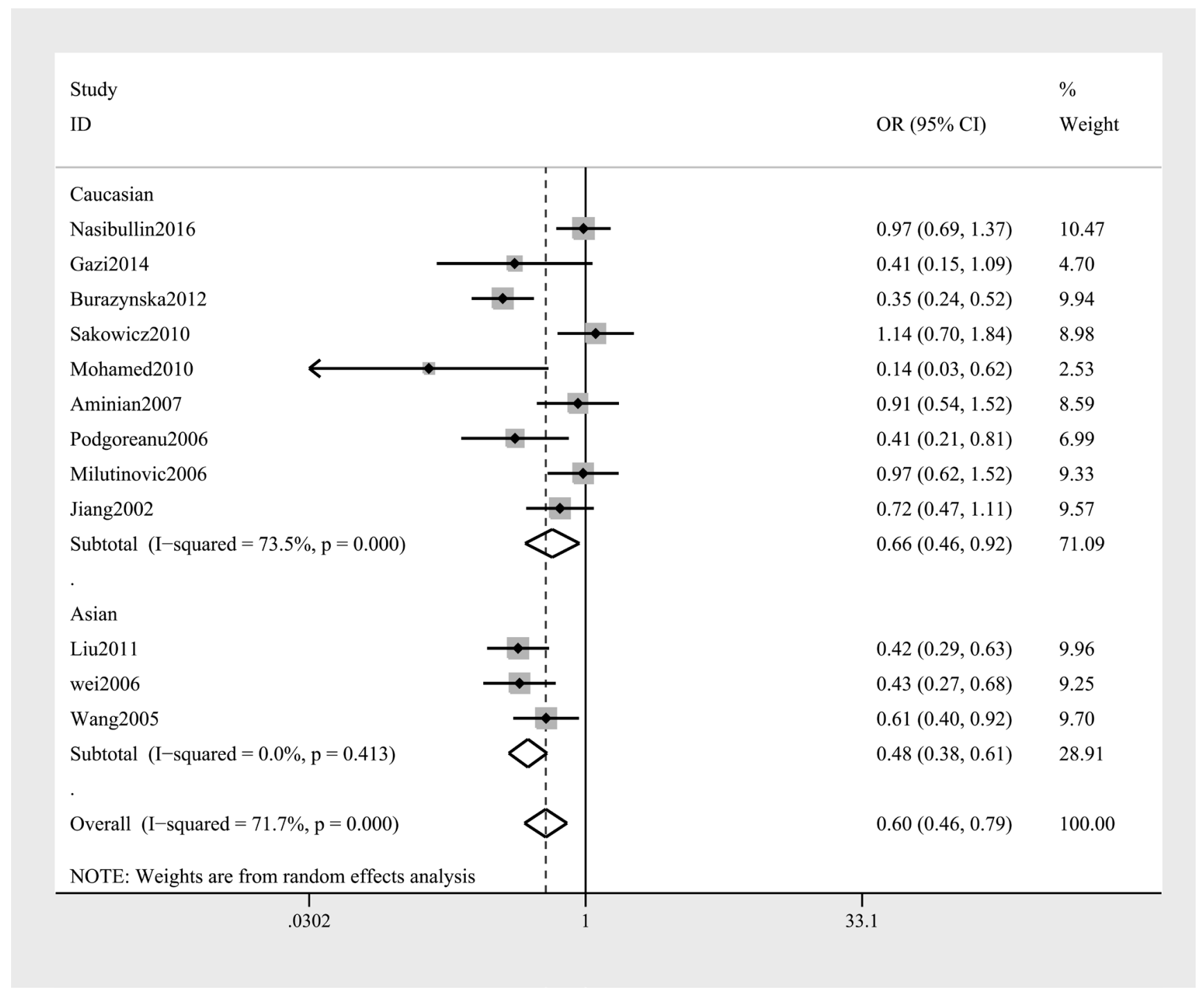

Figure 3: Stratification analysis of ethnicity shows odds ratio for the association between ICAM-1 gene rs5948 polymorphism and $\mathrm{MI}$ risk (AG+GG vs. AA). 
Table 3: Summary of the subgroup analyses in this meta-analysis

\begin{tabular}{|c|c|c|c|c|c|}
\hline Comparison & Category & Category & Studies & OR $(95 \%$ CI $)$ & $P$-value \\
\hline \multirow[t]{6}{*}{ Gvs. A } & Ethnicity & Caucasian & 8 & $0.61(0.44,0.84)$ & 0.003 \\
\hline & & Asian & 3 & $0.56(0.46,0.68)$ & $<0.001$ \\
\hline & SOC & HB & 10 & $0.60(0.46,0.79)$ & $<0.001$ \\
\hline & & PB & 1 & $0.59(0.44,0.78)$ & $<0.001$ \\
\hline & HWE & Yes & 10 & $0.60(0.46,0.79)$ & $<0.001$ \\
\hline & & No & 1 & $0.59(0.44,0.78)$ & $<0.001$ \\
\hline \multirow[t]{6}{*}{ AG+GG vs. AA } & Ethnicity & Caucasian & 9 & $0.66(0.46,0.92)$ & 0.016 \\
\hline & & Asian & 3 & $0.48(0.38,0.61)$ & $<0.001$ \\
\hline & SOC & HB & 10 & $0.55(0.41,0.74)$ & $<0.001$ \\
\hline & & $\mathrm{PB}$ & 2 & $0.89(0.57,1.39)$ & 0.614 \\
\hline & HWE & Yes & 11 & $0.55(0.41,0.74)$ & $<0.001$ \\
\hline & & No & 1 & $0.89(0.57,1.39)$ & 0.614 \\
\hline \multirow[t]{6}{*}{ GG vs. $A A+A G$} & Ethnicity & Caucasian & 8 & $0.51(0.31,0.82)$ & 0.006 \\
\hline & & Asian & 3 & $0.51(0.32,0.81)$ & 0.005 \\
\hline & SOC & HB & 10 & $0.53(0.35,0.80)$ & 0.002 \\
\hline & & $\mathrm{PB}$ & 1 & $0.39(0.25,0.61)$ & $<0.001$ \\
\hline & HWE & Yes & 10 & $0.53(0.35,0.80)$ & 0.002 \\
\hline & & No & 1 & $0.39(0.25,0.61)$ & $<0.001$ \\
\hline \multirow[t]{6}{*}{ GG vs. AA } & Ethnicity & Caucasian & 8 & $0.41(0.23,0.75)$ & 0.004 \\
\hline & & Asian & 3 & $0.38(0.23,0.61)$ & $<0.001$ \\
\hline & SOC & HB & 10 & $0.40(0.24,0.68)$ & 0.001 \\
\hline & & PB & 1 & $0.42(0.25,0.70)$ & 0.001 \\
\hline & HWE & Yes & 10 & $0.40(0.24,0.68)$ & 0.001 \\
\hline & & No & 1 & $0.42(0.25,0.70)$ & 0.001 \\
\hline \multirow[t]{6}{*}{ AG vs. AA } & Ethnicity & Caucasian & 8 & $0.75(0.57,1.00)$ & 0.046 \\
\hline & & Asian & 3 & $0.64(0.42,0.99)$ & $<0.001$ \\
\hline & SOC & HB & 10 & $0.62(0.49,0.78)$ & $<0.001$ \\
\hline & & $\mathrm{PB}$ & 1 & $1.13(0.69,1.82)$ & 0.631 \\
\hline & HWE & Yes & 10 & $0.62(0.49,0.78)$ & $<0.001$ \\
\hline & & No & 1 & $1.13(0.69,1.82)$ & 0.631 \\
\hline
\end{tabular}

SOC, source of controls; PB, population-based controls; HB, hospital-based controls.

However, the remaining studies failed to replicate this association $[11,13,15,19]$. Therefore, we thought it was necessary to perform a meta-analysis to yield a more accurate conclusion on the association between ICAM-1 gene rs5498 polymorphism and MI risk.

Several meta-analyses investigated the association between ICAM-1 rs5498 polymorphism and CAD before [26-29]. They all indicated that rs5498 polymorphism was a risk factor for CAD [26-29]. Among these metaanalyses, only one study by Li et al. found that ICAM-1 rs5498 polymorphism could increase the risk of MI [26].
In this meta-analysis, several inevitable defects should be considered [26]. First, two studies without related genotype data were included in MI group [22, 30]. Second, they did not include a Slovenian study [15], which actually met the inclusion criteria. Third, they did not perform the subgroup analyses of ethnicity, SOC and HWE among MI groups. To the best of our knowledge, this study is the first meta-analysis to explore the association between ICAM1 gene rs5498 polymorphism and MI risk. In this metaanalysis, we found rs5498 polymorphism was a protective factor for MI, which was different from the finding of $\mathrm{Li}$ 


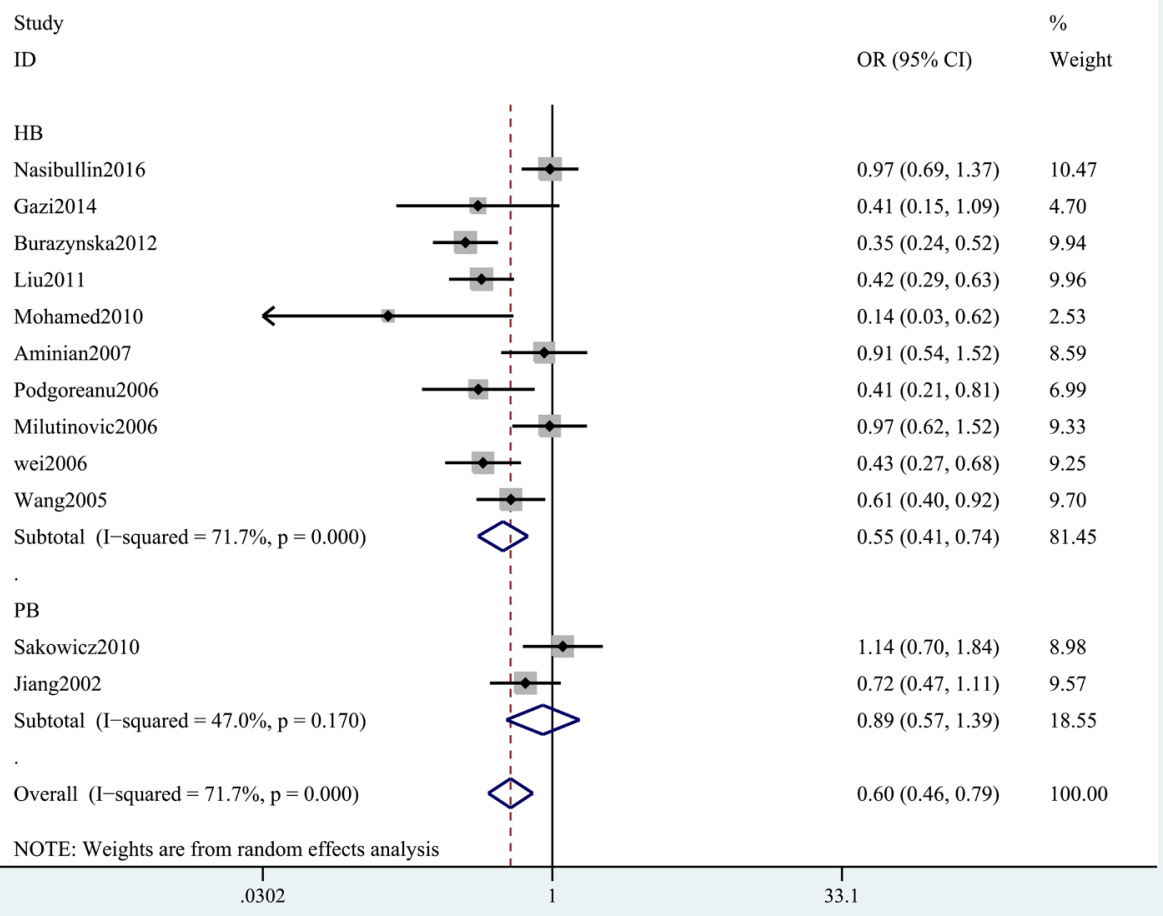

Figure 4: Stratification analysis of SOC shows odds ratio for the association between ICAM-1 gene rs5948 polymorphism and $\mathrm{MI}$ risk (AG+GG vs. $\mathrm{AA})$.

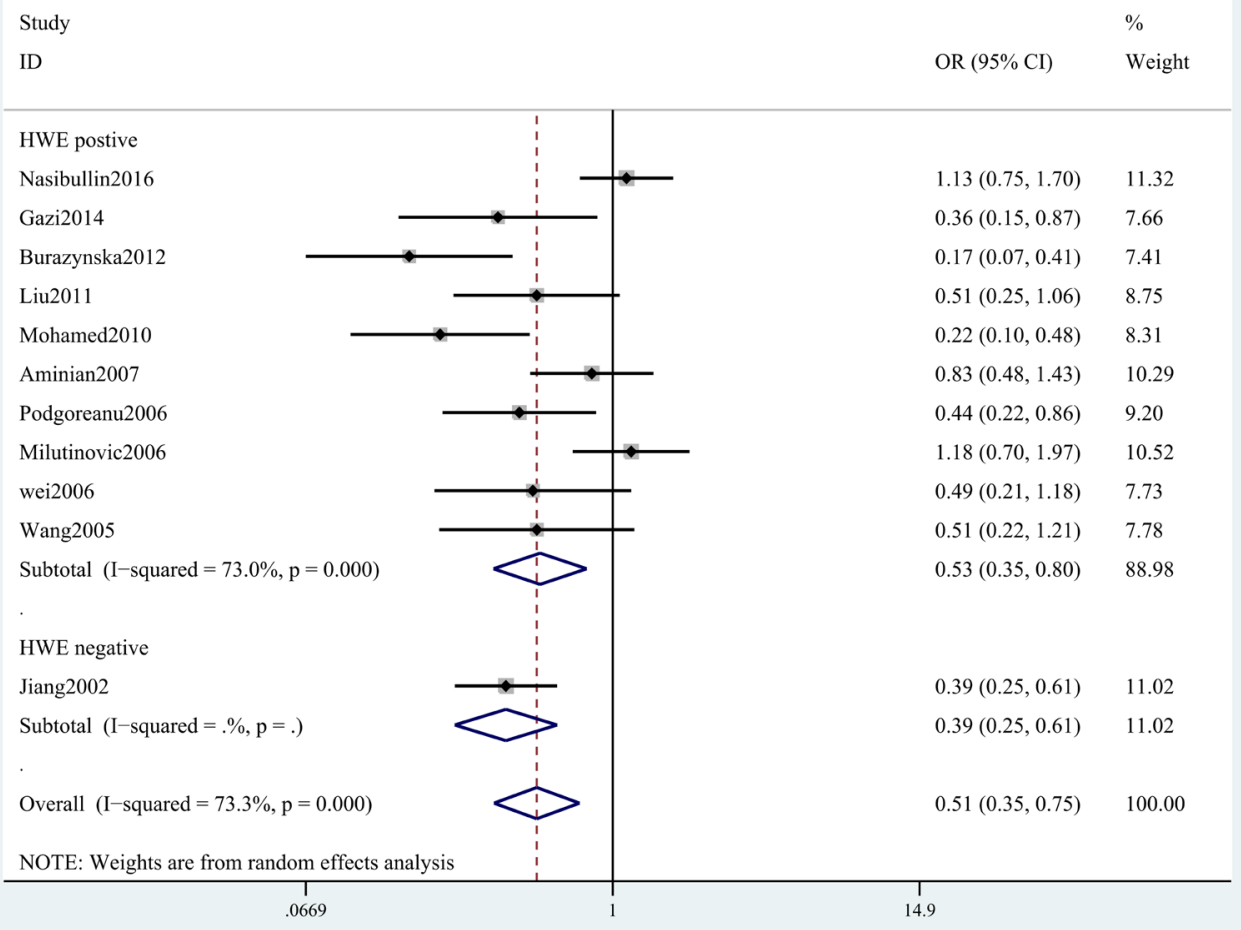

Figure 5: Stratification analysis of HWE status shows odds ratio for the association between ICAM-1 gene rs5948 polymorphism and MI risk (GG vs. AA+AG). 
et al. [26]. Stratification analysis of ethnicity also indicated that this polymorphism was associated with a decreased risk of MI among Caucasians and Asians. Obviously, this study did not obtain different results among different races. However, this meta-analysis only included Caucasian and Asian populations, but without other ethnicities such as Africans. Further studies among other ethnic populations are necessary to verify these findings. We thought our data were more robust than those of previous meta-analyses. More eligible studies were included in this meta-analysis $[13,15,17]$. In addition, the power analysis indicated that this meta-analysis had a power of $94.3 \%$ to detect the effect of rs5498 polymorphism on MI susceptibility with an OR of 0.60. Furthermore, subgroup analyses

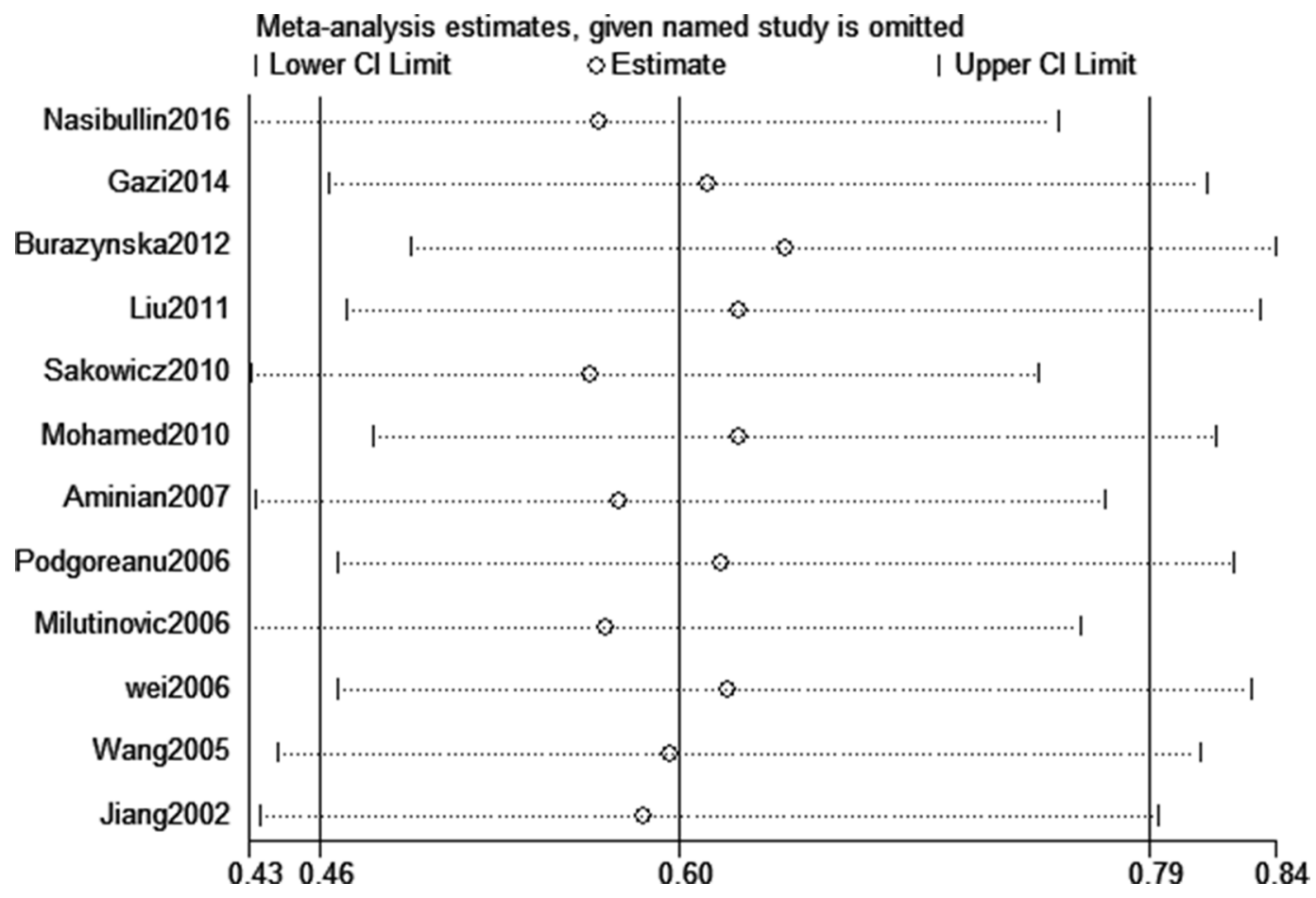

Figure 6: Sensitivity analysis for assessing the stability of the data of this meta-analysis (AG+GG vs. AA).

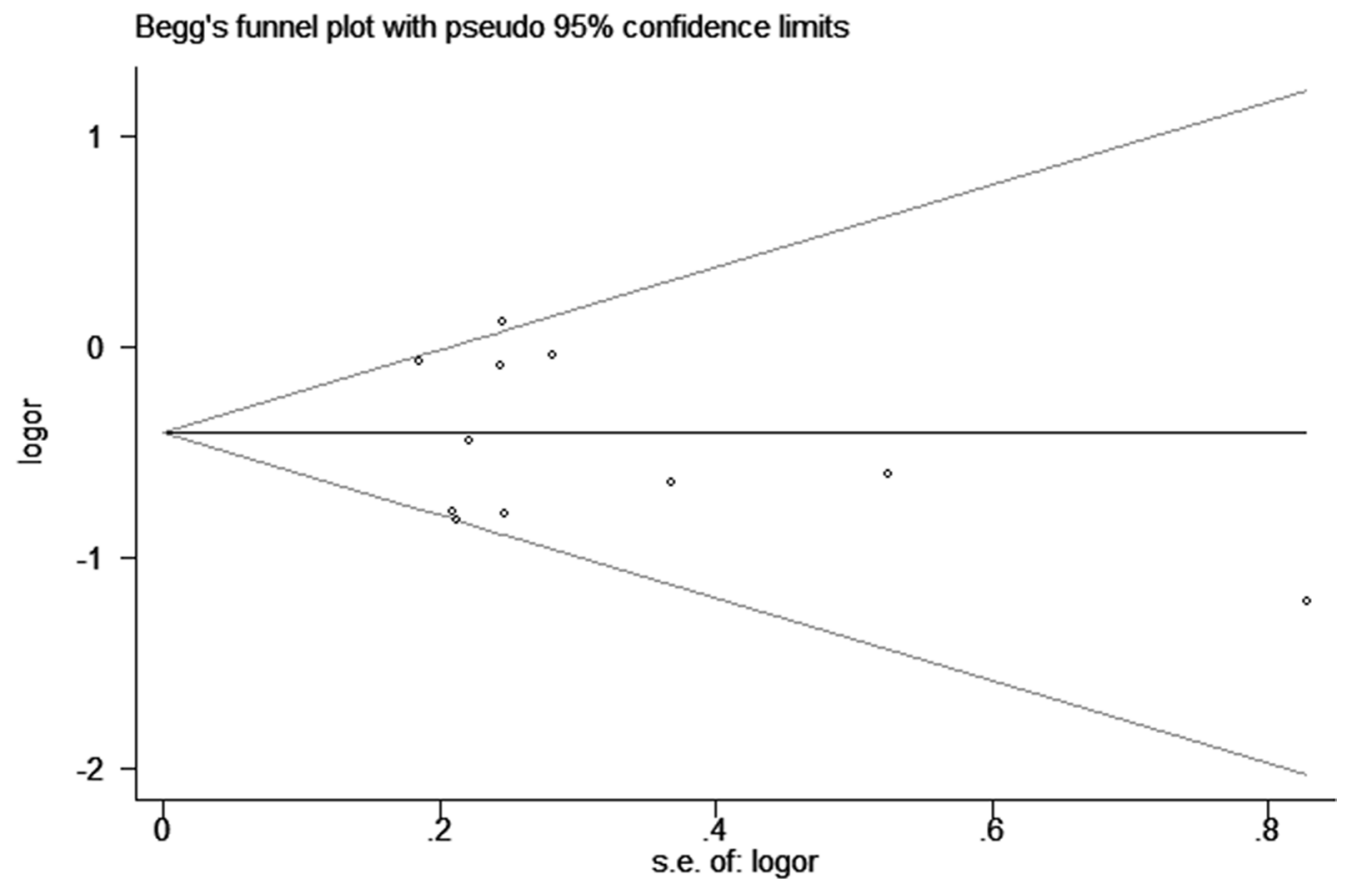

Figure 7: Begg's funnel plot of associated with ICAM-1 gene rs5948 polymorphism risk (AG vs. AA). 
of ethnicity, SOC and HWE also revealed this SNP was associated with the increased risk of MI. Finally, sensitivity analysis indicated that no single study could significantly alter the pooled ORs, suggesting that our results were reliable.

The potential limitations should be taken into consideration. First, MI is a multifactorial disease and may be affected by several confounding factors, such as History of MI, gender, and age. Thus, the function of a single SNP is limited. Second, sample size was relatively small and high between-study heterogeneity was observed in several genetic models. Third, only Caucasian and Asian populations were included in this meta-analysis and the results may be not applied to other ethnic groups. Fourth, the sample size of this meta-analysis was not very large. Fifth, the subgroup analysis might have insufficient statistical power to assess the real association. Sixth, we performed this meta-analysis with crude ORs since studies included in this meta-analysis lacked sufficient data for adjustment for confounding factors, which might affect the stability of our results.

In summary, our meta-analysis confirms that ICAM-1 gene rs5498 polymorphism is a protective factor for MI. Stratified analysis of ethnicity indicates that ICAM-1 rs5498 polymorphism is associated with the decreased risk of MI among Caucasians and Asians. Further epidemiological studies with larger simple sizes from different geographic regions are needed to confirm our findings.

\section{MATERIALS AND METHODS}

\section{Literature search}

Two investigators carried out a systematic electronic search independently in PubMed, EMBASE and China Knowledge Resource Integrated Database to identify relevant studies using the following key words: "Intercellular Adhesion Molecule-1," "ICAM1," "polymorphism," "single nucleotide polymorphism," "SNP" "Myocardial Infarction," "Cardiovascular Stroke," "Heart Attack," "Myocardial Infarct". No restrictions were placed on the search. Additional initially omitted studies (such as reference lists of identified studies) have been identified by hand screening.

\section{Inclusion and exclusion criteria}

Eligible studies met the following inclusion criteria: (1) evaluating the relationship between ICAM-1 rs5498 polymorphism and risk of MI; (2) sufficient data for calculating the pooled odds ratio (ORs) with 95\% confidence interval (CI); (3) case-control studies; (4) when studies had overlapping populations, the ones with the most complete data were included.

Exclusion criteria were as follows: 1) case-only studies; 2) meta-analysis or reviews; 3) studies that lacked detailed genotyping data; 4) duplicates of previous publications.

\section{Data extraction and quality assessment}

Two authors reviewed and extracted data independently in accordance with the inclusion criteria. From each study, the following information was extracted: first author's surname, publication year, country of origin, ethnicity, and numbers of cases and controls. Authors were contacted to provide supplemental data, if data were not available in the eligible studies. When studies included subjects of more than one ethnicity, genotype data were extracted separately.

Two authors independently assessed the quality of the selected studies using the Newcastle-Ottawa Scales (NOS) [31]. Total NOS scores ranged from 0 to 9. A score ranging 5 to 9 stars is considered to be a generally high methodological quality while a score ranging 0 to 4 is regarded as a relatively poor quality. The discrepancies were resolved by discussion or consulting with a third author.

\section{Statistical analysis}

All statistical analyses were performed using the Stata 11.0 software (StataCorp, College Station, TX, USA). Pooled ORs with corresponding 95\% CIs were calculated to evaluate the strength of association between ICAM-1 rs5498 polymorphism and risk of MI. Stratification analyses were carried out by ethnicity, SOC and HWE. $P<0.05$ was considered statistically significant. Taking possible between-study heterogeneity into consideration, we considered the presence of significant heterogeneity at the $10 \%$ level of significance and values of $\mathrm{I}^{2}$ exceeding $50 \%$ as an indicator of significant heterogeneity. When no heterogeneity was found with $P>0.10$ or $\mathrm{I}^{2}<50 \%$, a fixedeffect model was used. Otherwise, a random-effects model was applied [32]. Sensitivity analysis was conducted to determine the effect on the test of heterogeneity and evaluate the stability of the results by omitting each study in turn. Genotype distributions in the controls were tested for confirmation of Hardy-Weinberg equilibrium using the $\chi 2$ test. Publication bias was evaluated by visual inspection of symmetry of Begg's funnel plot and assessment of Egger's test [33]; $P<0.05$ was regarded as representative of statistical significance.

\section{Abbreviations}

CVD, cardiovascular disease; CAD, coronary artery disease; MI, myocardial infarction; AMI, acute myocardial infarction; ICAM-1, intercellular adhesion molecule 1; SOC, source of controls; HWE, Hardy-Weinberg equilibrium; CI, confidence interval; OR, odds ratio; NOS, Newcastle- Ottawa Scale; SNP, single nucleotide polymorphism. 


\section{ACKNOWLEDGMENTS AND FUNDING}

This work was supported by grants from Science Foundation of Health and Family Planning Commission of Zhejiang Province (2015KYB273, P.F. Hu) and the Natural Science Foundation of Zhejiang Province (Y13H020030, S.W. Huang).

\section{CONFLICTS OF INTEREST}

The authors declare no conflicts of interest.

\section{REFERENCES}

1. French KM, Somasuntharam I, Davis ME. Self-assembling peptide-based delivery of therapeutics for myocardial infarction. Adv Drug Deliv Rev. 2016; 96:40-53.

2. Goodacre S, Thokala P. The economics of cardiac biomarker testing in suspected myocardial infarction. Clin Biochem. 2015; 48:213-17.

3. Cummings DM, King DE, Mainous AG, Geesey ME. Combining serum biomarkers: the association of C-reactive protein, insulin sensitivity, and homocysteine with cardiovascular disease history in the general US population. Eur J Cardiovasc Prev Rehabil. 2006; 13:180-85.

4. Packard RR, Libby P. Inflammation in atherosclerosis: from vascular biology to biomarker discovery and risk prediction. Clin Chem. 2008; 54:24-38.

5. Ianni M, Callegari S, Rizzo A, Pastori P, Moruzzi P, Corradi D, Porcellini E, Campo G, Ferrari R, Ferrario MM, Bitonte S, Carbone I, Licastro F. Pro-inflammatory genetic profile and familiarity of acute myocardial infarction. Immun Ageing. 2012; 9:14.

6. Anbarasan C, Bavanilatha M, Latchumanadhas K, Ajit Mullasari S. ICAM-1 molecular mechanism and genome wide SNP's association studies. Indian Heart J. 2015; 67:282-87.

7. Benson V, McMahon AC, Lowe HC. ICAM-1 in acute myocardial infarction: a potential therapeutic target. Curr Mol Med. 2007; 7:219-27.

8. Wallén NH, Held C, Rehnqvist N, Hjemdahl P. Elevated serum intercellular adhesion molecule-1 and vascular adhesion molecule-1 among patients with stable angina pectoris who suffer cardiovascular death or non-fatal myocardial infarction. Eur Heart J. 1999; 20:1039-43.

9. Kamijikkoku S, Murohara T, Tayama S, Matsuyama K, Honda T, Ando M, Hayasaki K. Acute myocardial infarction and increased soluble intercellular adhesion molecule-1: a marker of vascular inflammation and a risk of early restenosis? Am Heart J. 1998; 136:231-36.

10. Niessen HW, Krijnen PA, Visser CA, Meijer CJ, Hack CE. Intercellular adhesion molecule-1 in the heart. Ann N Y Acad Sci. 2002; 973:573-85.
11. Sakowicz A, Fendler W, Lelonek M, Pietrucha T. Genetic variability and the risk of myocardial infarction in Poles under 45 years of age. Arch Med Sci. 2010; 6:160-67.

12. Podgoreanu MV, White WD, Morris RW, Mathew JP, Stafford-Smith M, Welsby IJ, Grocott HP, Milano CA, Newman MF, Schwinn DA, Perioperative Genetics and Safety Outcomes Study (PEGASUS) Investigative Team. Inflammatory gene polymorphisms and risk of postoperative myocardial infarction after cardiac surgery. Circulation. 2006; 114:I275-81.

13. Nasibullin TR, Timasheva YR, Sadikova RI, Tuktarova IA, Erdman VV, Nikolaeva IE, Sabo J, Kruzliak P, Mustafina OE. Genotype/allelic combinations as potential predictors of myocardial infarction. Mol Biol Rep. 2016; 43:11-16.

14. Mohamed AA, Rashed L, Amin H, Abu-Farha M, El Fadl SA, Pakhoum S. K469E polymorphism of the intercellular adhesion molecule-1 gene in Egyptians with coronary heart disease. Ann Saudi Med. 2010; 30:432-36.

15. Milutinović A, Petrovic D. The K469E polymorphism of the intracellular adhesion molecule 1 (ICAM-1) gene is not associated with myocardial infarction in Caucasians with type 2 diabetes. Folia Biol (Praha). 2006; 52:79-80.

16. Jiang H, Klein RM, Niederacher D, Du M, Marx R, Horlitz M, Boerrigter G, Lapp H, Scheffold T, Krakau I, Gülker H. C/T polymorphism of the intercellular adhesion molecule-1 gene (exon 6, codon 469). A risk factor for coronary heart disease and myocardial infarction. Int $\mathrm{J}$ Cardiol. 2002; 84:171-77.

17. Gazi E, Barutcu A, Altun B, Temiz A, Bekler A, Urfali M, Silan F, Colkesen Y, Ozdemir O. Intercellular adhesion molecule-1 K469E and angiotensinogen T207M polymorphisms in coronary slow flow. Med Princ Pract. 2014; 23:346-50.

18. Buraczynska M, Zaluska W, Baranowicz-Gaszczyk I, Buraczynska K, Niemczyk E, Ksiazek A. The intercellular adhesion molecule-1 (ICAM-1) gene polymorphism $\mathrm{K} 469 \mathrm{E}$ in end-stage renal disease patients with cardiovascular disease. Hum Immunol. 2012; 73:824-28.

19. Aminian B, Abdi Ardekani AR, Arandi N. ICAM-1 polymorphisms (G241R, K469E), in coronary artery disease and myocardial infarction. Iran J Immunol. 2007; 4:227-35.

20. Wei YS, Tang RG, Yuan XH, Pan Y, Lan J. [Association between polymorphism of intercellular adhesion molecule-1 gene K469E and coronary heart disease]. [Article in Chinese]. Zhongguo Mian Yi Xue Za Zhi. 2006; 22:1056-59.

21. Wang M, Li Y, Zhang PA, Yang C, Xiang P, Wei Y. [Study on the intercellular adhesion molecule-1 gene polymorphisms in a Chinese population with myocardial infarction]. [Article in Chinese]. Zhonghua Liu Xing Bing Xue Za Zhi. 2005; 26:702-6. 
22. Liu Z, Wei Y, Tan Z. [Relationship between intercellular adhesion molecule-1 K469E gene polymorphism and coronary heart disease in Zhuang]. [Article in Chinese]. Zhongguo Laonianxue Zazhi. 2011; 30:581-589.

23. Usami Y, Ishida K, Sato S, Kishino M, Kiryu M, Ogawa Y, Okura M, Fukuda Y, Toyosawa S. Intercellular adhesion molecule-1 (ICAM-1) expression correlates with oral cancer progression and induces macrophage/cancer cell adhesion. Int J Cancer. 2013; 133:568-78.

24. Ley K, Miller YI, Hedrick CC. Monocyte and macrophage dynamics during atherogenesis. Arterioscler Thromb Vasc Biol. 2011; 31:1506-16.

25. Lawson C, Wolf S. ICAM-1 signaling in endothelial cells. Pharmacol Rep. 2009; 61:22-32.

26. Li D, Qu C, Dong P. The ICAM-1 K469E polymorphism is associated with the risk of coronary artery disease: a meta-analysis. Coron Artery Dis. 2014; 25:665-70.

27. Yanyan L. Intercellular adhesion molecule-1 E469K gene polymorphism and coronary artery disease in the Chinese population: a meta-analysis involving 3065 subjects. Clin Cardiol. 2012; 35:55-60.
28. Ji YN, Wang Q, Zhan P. Intercellular adhesion molecule 1 gene K469E polymorphism is associated with coronary heart disease risk: a meta-analysis involving 12 studies. Mol Biol Rep. 2012; 39:6043-48.

29. Zou S, Pan X, Chen Z, Wei C, He B, Zhang H. Intercellular adhesion molecule-1 K469E polymorphism and risk of coronary artery disease: a meta-analysis. Med Sci Monit. 2014; 20:2677-82.

30. Shang Q, Lu FH, Wen PE, Wu F, Zhao Y, Su G, Tian Q. The study of intercellular adhesion molecule-1 gene polymorphisms C469T in elderly patients with coronary heart disease. Chin J Geriatr. 2005; 24:444-45.

31. Stang A. Critical evaluation of the Newcastle-Ottawa scale for the assessment of the quality of nonrandomized studies in meta-analyses. Eur J Epidemiol. 2010; 25:603-05.

32. Higgins JP, Thompson SG. Quantifying heterogeneity in a meta-analysis. Stat Med. 2002; 21:1539-58.

33. Peters JL, Sutton AJ, Jones DR, Abrams KR, Rushton L. Comparison of two methods to detect publication bias in meta-analysis. JAMA. 2006; 295:676-80. 